\title{
CIENCIAS DEL COMPORTAMIENTO Y GOBERNANZA COLABORATIVA: EXPERIENCIAS DE CULTURA CIUDADANA EN MEDELLÍN, COLOMBIA
}

\author{
BEHAVIOURAL SCIENCES AND COLLABORATIVE GOVERNANCE: EXPERIENCES OF \\ CIVIC CULTURE IN MEDELLÍN, COLOMBIA
}

\author{
Jonathan Echeverri Álvarez, jechev39@eafit.edu.co \\ Laura Gallego Moscoso, lgalleg5 @ eafit.edu.co \\ Santiago Silva Jaramillo, ssilvaja@eafit.edu.co
}

Centro de Análisis Político, Universidad EAFIT

\begin{abstract}
Resumen: Este artículo realiza una síntesis de dos experiencias representativas en ciencias del comportamiento e intervenciones públicas por parte de la Alcaldía de Medellín, Colombia (periodo 2016-2019) y el Centro de Análisis Político de la Universidad EAFIT. Estas experiencias constituyen estrategias de intervención pública para el cambio de comportamiento en direcciones que mejoran la confianza y la convivencia entre los ciudadanos. El texto presenta algunas consideraciones sobre las ciencias del comportamiento aplicadas al diseño, implementación y evaluación de intervenciones públicas. Luego, reseña el Laboratorio de Cultura Ciudadana como un espacio de gestión del conocimiento y gobernanza colaborativa. Finalmente, se presenta Ciudadanos como Vos como una intervención pública que busca generar vínculos de confianza a través de la visibilización de comportamientos pro-sociales.
\end{abstract}

Palabras clave: Ciencias del comportamiento, gobernanza colaborativa, cultura ciudadana, innovación social, experimentación, normas sociales.

\begin{abstract}
This article synthesises two representative experiences in behavioural science and public interventions by the Mayor's Office of Medellin, Colombia, from 2016 to 2019 and the Centre for Political Analysis at EAFIT University. The experiences involved public intervention strategies for behavioural change in directions that improve trust and coexistence among citizens. The article presents some considerations on the behavioural sciences applied to the design, implementation and evaluation of public interventions. Thereafter, it reviews the Citizen Culture Laboratory as a space for knowledge management and collaborative governance. Last, it presents "Citizens like you" as a site of public intervention that seeks to generate bonds of trust through the visibility of prosocial behaviours.
\end{abstract}

Keywords: Behaviour science, collaborative governance, civic culture, social innovation, experimentation, social standards.

\section{Introducción}

La ciudad de Medellín ha tenido como una de sus preocupaciones más recientes la pregunta sobre cómo fortalecer las instituciones locales y propiciar un diálogo permanente con sus habitantes. Medellín, que hasta hace pocos años enfrentaba una crisis de violencia incapaz de ser gestionada ante la debilidad de su entramado institucional, ha tenido que hacerle frente a enormes desafíos poniendo en el centro de su respuesta la acción colectiva y la cultura ciudadana, el rol de los ciudadanos para impulsar y crear mejores soluciones a los desafíos colectivos. En este proceso de cambio que ha

\begin{tabular}{|c|c|}
\hline $\begin{array}{l}\text { EUROPEAN } \\
\text { PUBLIC \&OCIAL } \\
\text { INNOVATION }\end{array}$ & $\begin{array}{c}\text { European Public \& Social Innovation Review } \\
\text { Volume 4, Issue 1, } 2019 \text { ISSN 2529-9824 } \\
\text { Creative Commons 4.0. International }\end{array}$ \\
\hline
\end{tabular}


vivido la ciudad, se ha cuestionado el rol pasivo de la sociedad civil en la toma de decisiones públicas y se han impulsado desde el sector público, privado y social espacios de diálogo, participación y corresponsabilidad en los que se ven involucrados para la identificación, comprensión e intervención de problemas públicos.

Desde esta perspectiva, han venido consolidándose varias experiencias e iniciativas que ponen en el centro de la reflexión la necesidad de propiciar desde el gobierno local el encuentro ciudadano, el diálogo social y la experimentación alrededor de los retos y las oportunidades que representa la cultura ciudadana y las ciencias del comportamiento en Medellín. Iniciativas que se proponen motivar transformaciones positivas hacia la convivencia desde el empoderamiento y la corresponsabilidad ciudadana, promueven intervenciones de espacios públicos, diseño de estrategias públicas, construcción de procesos con las comunidades en los territorios, el reconocimiento y la movilización entre la ciudadanía; así como la cocreación y cartografías subjetivas que enriquecen las miradas y voces de los ciudadanos para responder a la complejidad del orden social.

Este artículo intenta recoger algunas de las reflexiones y experiencias que la ciudad de Medellín, a partir del liderazgo de la Secretaría de Cultura y con el acompañamiento de la Universidad EAFIT, ha desarrollado para promover la confianza entre las instituciones y las personas, promover el cambio comportamental y el cumplimiento de normas y acuerdos; y abrir espacios para la conversación y la deliberación ciudadana en torno a los retos de lo público y a la capacidad limitada que tiene el Estado local para resolverlos.

Así, teniendo las ciencias del comportamiento y la cultura ciudadana como enfoques para el diseño de intervenciones públicas, se destacan dos de las iniciativas que se han venido impulsando en Medellín, el Laboratorio de Cultura Ciudadana y Ciudadanos como Vos, para involucrar al ciudadano en el diseño de alternativas de solución a los desafíos públicos. En un esfuerzo por recoger, estudiar y encontrar aprendizajes sociales en algunos de los programas y estrategias de Medellín en su agenda de construcción de ciudadanía, este artículo intenta evidenciar cómo el capital social de una ciudad como Medellín se construye desde la capacidad que tienen las instituciones públicas por hacer corresponsables a los habitantes de su ciudad, a los actores públicos, privados y a la academia en el reconocimiento y la transformación de imaginarios, comportamientos y acciones (Silva, 2017).

\section{Ciencias del comportamiento en intervenciones públicas}

El uso de las ciencias del comportamiento para el diseño, implementación y evaluación de intervenciones públicas, con frecuencia, permite abrir ventanas de oportunidad, formar ciudadanos en el ejercicio de su autonomía política, y realizar investigación de manera permanente para redefinir y volver a diagnosticar con las intervenciones. Esta perspectiva pretende el cambio de comportamiento, individual y colectivo, en direcciones deseables que mejoren la calidad de vida y el bienestar de las personas. Este enfoque pone como centro de gravedad la decisión humana y concibe estrategias de cambio como el uso de nudges (pequeños empujes); boost (formación de autonomía); y el diagnóstico e intervención de normas sociales (Bicchieri, 2018; Hertwig \& GrüneYanoff, 2017; Thaler \& Sunstein, 2017). En estos usos es fundamental el respeto por la libertad humana, es decir, se proponen cambios en direcciones deseables sin restricciones notables a las decisiones de las personas (Sunstein, 2017). Por tal razón, la gobernanza colaborativa, entendida como un espacio de comunicación abierta entre gobierno y ciudadanía, permite discutir y evaluar los cambios de comportamientos sugeridos en comunidades concretas. En la intervención pública es crucial entonces tener un espacio abierto de comunicación y concebir estrategias de cambio sobre la base de investigaciones del comportamiento humano. De acuerdo con el Grupo Banco Mundial (2015, p. 5):

"Las investigaciones de los últimos tiempos nos han permitido conocer mejor las influencias psicológicas, sociales y culturales a que están sujetas la toma de decisiones y la conducta de los seres humanos, y han demostrado que dichas influencias tienen un impacto significativo en los resultados en términos de desarrollo. (...) Las investigaciones revelan que las pequeñas diferencias de contexto, conveniencia e importancia tienen grandes efectos en las opciones cruciales, como enviar a un niño a la escuela, prevenir una enfermedad o ahorrar para iniciar una actividad comercial. Esto significa que los especialistas en el desarrollo deben centrarse no solo en determinar qué intervenciones son necesarias sino también en cómo se ponen en práctica". 
Esta consideración sobre las pequeñas diferencias de contexto ha motivado la generación de conocimiento con intervenciones en áreas como la salud, el empleo, la pobreza, las finanzas personales, el desarrollo cognitivo infantil, la contaminación y cambio climático, entre otros escenarios de interés en relación con la toma de decisiones. Thaler y Sunstein (2017, p. 13) proponen el concepto de nudge, palabra que en el idioma inglés significa "Empujar suavemente o dar un golpecito en las costillas, sobre todo con el codo a fin de avisar, recordar o amonestar suavemente a otro". El nudge en el ámbito de la elección implica estimular, incentivar o encaminar en la toma de decisiones. Ambos autores postulan que "los humanos también responden a los incentivos, pero se ven igualmente influidos por los nudges. Desplegando adecuadamente tanto incentivos como nudges, podemos aumentar nuestra capacidad para mejorar la vida de la gente y contribuir a resolver muchos de los grandes problemas de la sociedad" (Sunstein, 2017, p. 23). Estas ideas invitan a pensar que el bienestar y el desarrollo humano se ve influenciado por las decisiones que toman los individuos y también por las oportunidades de elección que se ofrecen en los contextos públicos, a través por ejemplo de intervenciones para la ciudadanía (Kahneman, 2012; Grupo Banco Mundial, 2015; Thaler \& Sunstein, 2017).

Existen algunas experiencias internacionales de política pública en esta dirección. Por ejemplo, la propuesta de David Cameron con The Nudge Unit, en el año 2010 y la agencia pública de seguimiento a políticas públicas de Cultura y Cambio de Comportamiento Carnegie UK Trust. En el primer caso se incorporó también una agencia de investigación para hacer seguimiento a las intervenciones diseñadas. Las primeras dos intervenciones valiosas refieren a la disminución de evasión de impuestos y al cumplimiento de normas de tránsito. Esto a través de pequeños cambios de contexto e información para dirigir la acción colectiva y las decisiones de las personas en comportamientos que mejoren sus vidas. Esta experiencia positiva en los hallazgos luego comenzó a replicarse a nivel mundial en el diseño e implementación de políticas públicas. La agencia Carnegie UK Trust, con el plan de intervención diseñado durante el 2016 y 2020, se concentra en mejorar el bienestar de la población británica en general e incorpora el diseño e intervención sobre confianza y cumplimiento de acuerdos abordando el uso de tecnologías digitales. Es decir, la implementación del Plan incluye trabajo de campo e investigación en Redes Sociales Digitales.

También es posible resaltar, en la Administración Obama, el grupo denominado The Social and Behavioral Sciences Team (SBST). Liderado por Cass Sunstein, con la participación de investigadores provenientes de la ciencia política, el derecho y la economía del comportamiento. Luego de los resultados positivos obtenidos durante el 2014, en investigación e intervención, se emite una Orden Federal en septiembre 15 de 2015 para utilizar las Ciencias del Comportamiento al servicio de la población en general. Los dos últimos reportes publicados disponibles del año 2015 y 2016, señalan el desarrollo de un espacio interactivo denominado Community Action Deck. Este espacio sirvió para conectar en diálogo a funcionarios públicos, ciudadanía e investigadores académicos. Un diálogo para desarrollar intervenciones en diversos ámbitos como cambio climático; discusión de la reforma de justicia criminal; mejorar la eficiencia y efectividad del Gobierno en el desarrollo de sus intervenciones; entre otros. Desde esta perspectiva, la conversación interinstitucional y transdisciplinar es fundamental para incentivar la confianza ciudadana y el cumplimiento de acuerdos, normas sociales y disposiciones legales.

En Medellín, Colombia, entre la última administración de la Alcaldía de Medellín, específicamente con la Subsecretaría de Cultura Ciudadana, el Laboratorio de Cultura Ciudadana y el Centro de Análisis Político de la Universidad EAFIT, se han desarrollado una serie de estrategias de intervención para el cambio de comportamiento que permita la confianza, cooperación, cohesión social, cumplimiento de acuerdos y disposición a obedecer la ley entre los ciudadanos. Esto con una estrategia de gobernanza colaborativa y un componente de gestión del conocimiento para hacer seguimiento, con evidencia empírica, a las diversas intervenciones implementadas. En este proceso se han realizado tres publicaciones académicas que dan cuenta de la experiencia. La primera realizada en el año 2016 con el título Pensar y construir el territorio desde la cultura. Derecho a la ciudad, cultura ciudadana e innovación pública (Tamayo, 2016). La segunda del año 2017 titulada Imaginarios comunes, sueños colectivos y acciones ciudadanas: Pensando Medellín en clave de cultura ciudadana, derecho a la ciudad e innovación pública (Tamayo, 2017). Y finalmente, la tercera publicación del año 2018 titulada Cultura ciudadana. Reflexiones y 
experiencias de ciudad (Echeverri \& Gallego, 2019). Estos tres ejercicios son el resultado de un trabajo colaborativo entre funcionarios públicos, investigadores y ciudadanía. Y han pretendido “(...) que las lecciones propias puedan resultar de relevancia para otras personas, entidades u organizaciones con retos similares y con intención de abordarlas desde el enfoque de cultura ciudadana y economía comportamental" (Echeverri et al., 2019, p. 18). A continuación, se presentan un par de intervenciones representativas en esta dirección.

\section{Experiencias}

\subsection{Cocreación ciudadana y cambio social: el Laboratorio de Cultura Ciudadana de Medellín}

El Laboratorio de Cultura Ciudadana es una plataforma para la gestión del conocimiento aplicado que nace en 2016, orientada a motivar el cambio social y comportamental desde la interacción, conversación y colaboración con los ciudadanos de Medellín en torno a los desafíos colectivos de lo público. En un esfuerzo por involucrar desde el gobierno local al sector privado y social, y acompañado de la investigación académica de la Universidad EAFIT, el Laboratorio se ha convertido en una experiencia que intenta poner en la agenda pública de la ciudad la necesidad de reflexionar, idear y experimentar con las reglas, los contextos y los comportamientos asociados a la construcción de ciudadanía en Medellín (Silva, 2017).

Esta plataforma ha sido la materialización de un propósito trazado por el Plan de Desarrollo Municipal 2016-2019, "Medellín Cuenta con Vos" y su intención por hacer de la cultura ciudadana un eje trasversal a los programas y acciones de la administración, e incidir en la transformación de los problemas públicos desde el rol y los efectos que sobre ellos tienen las prácticas ciudadanas. Busca propiciar espacios de diálogo para la construcción de soluciones a los principales retos de la ciudad y fortalecer el compromiso con la ciudadanía para la comprensión e intervención de los mismos, con el fin de construir confianza entre las instituciones del Estado y los habitantes de Medellín, además de promover el cumplimiento de normas, la generación de acuerdos sobre aquellos asuntos determinantes para la preservación del orden social (Echeverri \&
Gallego, 2019), y la identificación y comprensión de imaginarios, narrativas y valores de los ciudadanos frente a la acción pública.

La reflexión y el diálogo que desde la academia ha propiciado el Laboratorio, la gestión del conocimiento y la generación de conversaciones entre diversos actores convierten a esta iniciativa en una experiencia que permite articular y hacer transversal las soluciones a problemáticas, necesidades y retos de la ciudad con una visión holística, transectorial y multidisciplinar, integrando metodologías de diseño y experiencia ciudadana. Desde lo metodológico, ha logrado posicionarse como un espacio de cocreación de servicios públicos y capacidades institucionales conectados con la experiencia de la ciudadanía y en función de la generación de respuestas integrales a sus necesidades más cotidianas.

Como escenario de diálogo, ha permitido el intercambio de conocimientos, la construcción colectiva de soluciones a problemas públicos y el fortalecimiento del capital social de la ciudad. En otras palabras, es un espacio para la construcción de cultura cívica y la reivindicación de la confianza, el cumplimiento de las normas y el establecimiento de redes en lo público.

La innovación pública, la cultura ciudadana, la gobernanza colaborativa, la economía comportamental y la experimentación han sido conceptos que han permitido orientar y consolidar el trabajo metodológico del Laboratorio de Cultura Ciudadana. En su propósito por cómo lograr que el ciudadano cumpla las reglas, los acuerdos y confíe en los demás (Mockus, 2002), el Laboratorio ha logrado la identificación y transformación de comportamientos colectivos en la ciudad desde las herramientas que este marco teórico le ha proporcionado. En este camino, la Universidad EAFIT ha contribuido a través del Modelo Experimental de Corresponsabilidad Ciudadana (CIVICCO), una metodología que pone en el centro del desarrollo de alternativas de solución a los problemas públicos al ciudadano, motivando la creación colectiva de respuestas a esos retos desde la corresponsabilidad entre actores (organizaciones, personas o instituciones), e integrando cuatro componentes necesarios para el proceso de creación y fortalecimiento del capital social: la Plataforma de Innovación Pública como fundamento teórico, metodológico y operativo para el desarrollo del Laboratorio; la diversidad y el rol de los actores que participan de la experiencia; la conceptualización de la experiencia de creación colectiva a través del diagnóstico, el diseño de la experiencia, el 
desarrollo de la experiencia y el producto; y, un cuarto componente de acompañamiento en procesos de innovación social, que pretende hacer sostenible el fortalecimiento del capital social (Echeverri \& Londoño, 2017).

A partir de este modelo, el Laboratorio Social concibe la cultura como una dimensión mediadora, a partir de la cual la interacción entre diferentes actores sociales construye y transforma las realidades simbólicas y comportamentales en un contexto particular. Así, CIVICCO se convierte en una herramienta para la comprensión, el diseño y la validación de ideas y experiencias creativas colaborativas dirigidas a la acción, la participación ciudadana, la generación de confianza interpersonal y la promoción de la convivencia. El Laboratorio de Cultura Ciudadana se concibe como un resultado de dicho modelo, aplicado por la Alcaldía de Medellín y enfocado en hacer de la cultura ciudadana, la innovación pública, la experimentación y el estudio de los comportamientos ciudadanos, herramientas en el diseño de los instrumentos de intervención pública, del gobierno, a los retos de la ciudad y toma de decisiones para solucionarlos.

En este sentido, el Laboratorio de Cultura Ciudadana se desarrolla de manera simultánea en cuatro dimensiones: (1) gestión de conocimiento, (2) cocreación sectorial con servidores públicos, (3) experimentación y cocreación con ciudadanos; (4) y, articulación y transferencia de capacidades en la administración pública para la integración de soluciones a los problemas públicos.

\subsubsection{La gestión del conocimiento}

Se trata de la dimensión que consolida y orienta la plataforma en términos metodológicos. Constituye un componente transversal que se desarrolla a partir del estudio de las ciencias sociales, llevando a cabo investigaciones aplicadas con el fin de orientar y articular la reflexión, el diseño y la implementación de estrategias de intervención con una perspectiva de análisis comparado y multidisciplinar. Desde esta dimensión, se trabajan los conceptos anteriormente mencionados y las herramientas que soportan el hacer de la iniciativa, a saber, la innovación pública, la cultura ciudadana, la gobernanza colaborativa y la experimentación. Brevemente, se entiende por ello:

i) Innovación pública: la innovación en lo público entendida como herramienta que le permite al gobierno adaptarse a los cambios, diseñando y gestionando servicios públicos centrados en la experiencia de los ciudadanos.

Así, la innovación pública es un enfoque experimentación y diseño que promueve la mejora continua de los servicios públicos para impactar positivamente la calidad de vida de la ciudadanía (Sánchez et al., 2013).

ii) La cultura ciudadana: uno de los principales enfoques del trabajo del Laboratorio ha sido la cultura ciudadana, entendida como un acumulado de procesos que facilitan el derecho a la ciudad y permiten que los ciudadanos construyan lo público a partir de reflexiones simbólicas y culturales, y fortalezcan el capital social (Tamayo, 2016). El Laboratorio se aproxima a la cultura ciudadana a partir de lo expuesto por el profesor Antanas Mockus y el equipo de la Corporación Visionarios por Colombia -Corpovisionarios-, que han promovido el enfoque de políticas públicas de cultura ciudadana; así como, el enfoque del derecho a la ciudad, para ir más allá de los imaginarios, valores y costumbres asociados a la convivencia y reivindicar los derechos de los ciudadanos en sus comunidades y territorios para la construcción colectiva de lo público. En sintonía con los aportes que ha supuesto para el Laboratorio integrar como enfoque conceptual y metodológico la cultura ciudadana, la economía conductual también ha sido una nueva aproximación a la agenda de gestión del conocimiento de la plataforma. El enfoque de la economía conductual, preocupada por explicar la naturaleza de la decisión humana en condiciones de riesgo e incertidumbre, le ha permitido al Laboratorio reflexionar en torno al comportamiento individual y colectivo de los ciudadanos, y diseñar intervenciones que permitan integrar dichos comportamientos y decisiones asociadas para mejorar la calidad de vida de las personas y orientar el cambio de imaginarios y conductas en favor de la convivencia. 
Figure 1. Modelo Experimental de Corresponsabilidad Ciudadana (CIVICCO)

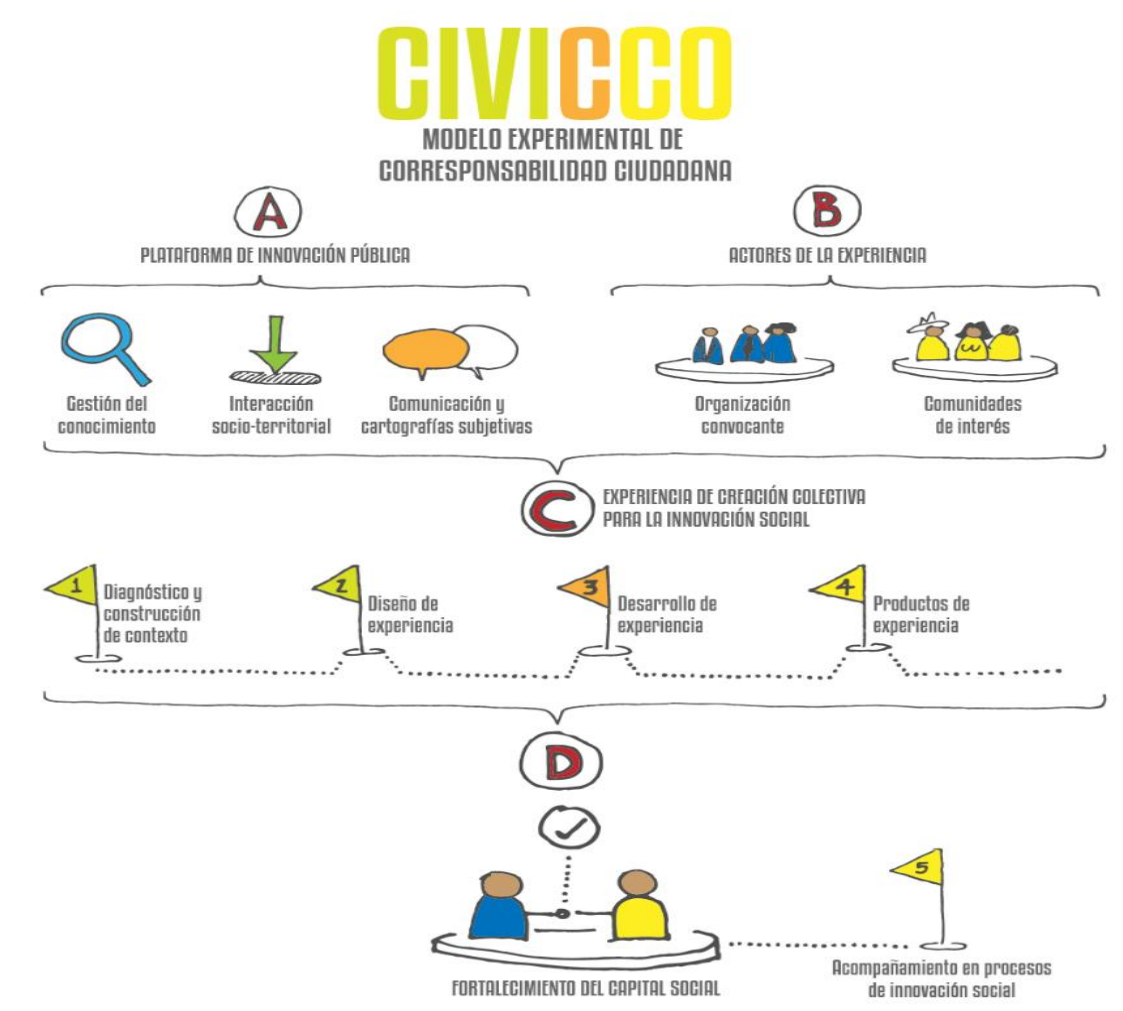

Fuente: Centro de Análisis Político, Universidad EAFIT.

iii) La gobernanza colaborativa: al ser la corresponsabilidad y el diálogo plural dos apuestas del Laboratorio, este se ha constituido en una experiencia para la generación de acuerdos y la participación en la toma de decisiones públicas, es decir, en una plataforma para facilitar la gobernanza colaborativa (Ansell \& Gash, 2007). La gobernanza colaborativa entendida como la definición de arreglos cooperativos entre actores sociales, públicos y privados en la ciudad con el propósito de abordar los desafíos de la misma, de manera coordinada y conjunta. Siendo el Laboratorio de Cultura Ciudadana una apuesta institucional por la construcción de confianza entre ciudadanía y Estado en clave de cultura ciudadana, innovación pública y fortalecimiento del capital social, la gobernanza colaborativa se convierte en un principio de acción que ordena la forma cómo la Alcaldía de Medellín, la Secretaría de Cultura y el Laboratorio interactúan con diversos actores de ciudad y logran impactos mayores y efectivos frente a retos sociales de la agenda pública.

iv) La experimentación, cocreación y cartografías sociales: por último, el Laboratorio de Cultura Ciudadana integra en su hacer la experimentación, la cocreación y las cartografías sociales como herramientas para tramitar las demandas ciudadanas y ordenar la capacidad institucional para darles respuesta (Herrera, 2008). A través de ellas, le facilitan la construcción colectiva de productos, servicios, procesos, o acciones que generan valor para la sociedad (Bason, 2013, p. 6); esto es, una plataforma en la que las soluciones son diseñadas con las personas, se construyen lazos y compromisos para su posterior desarrollo, y se hace corresponsable a la ciudadanía del valor de las mismas en el orden social. 


\subsubsection{Cocreación sectorial con servidores públicos}

Para asegurar la transferencia de herramientas tomadas de la innovación pública, la cocreación y la experimentación, esto es, los resultados del proceso de gestión del conocimiento a los servidores públicos del municipio de Medellín, se concibe la dimensión de cocreación sectorial con servidores en el Laboratorio. Se propone ser el medio a través del cual el servidor público adquiere nuevas capacidades y fortalece las herramientas con las que cuenta para entablar la comunicación con otros actores y la aplicación de nuevas metodologías para el diseño de instrumentos de gestión para los problemas públicos.

\subsubsection{Cocreación y corresponsabilidad ciudadana}

Desde la dimensión de corresponsabilidad ciudadana, es el habitante común de Medellín un aliado en la identificación y el diseño de retos colectivos para mejorar la calidad y la convivencia en la ciudad. Desde el Laboratorio, esta dimensión se propone ofrecer herramientas para que los ciudadanos se vinculen en la creación de soluciones, a los problemas que se enfrentan en su cotidianidad y permitan fortalecer $y$ hacer efectivas las acciones de gobierno. En dicho proceso, el Laboratorio ha desarrollado una serie de metodologías orientadas a generar procesos de colaboración, interacción y corresponsabilidad. Por ejemplo, a través de juegos ciudadanos a partir de la conjunción de elementos de experimentación y juegos económicos como modelos que escenifican situaciones de posibles conflictos o cooperación, en las que se pueden identificar escenarios y pautas que se repiten con frecuencia en el mundo real $y$ ofrecen pistas para intervenciones y acciones de transformación social. Los experimentos han permitido identificar y recolectar en el plano subjetivo información sobre cómo los ciudadanos se perciben en su relación con las instituciones, con los territorios y entre ellos mismos ante los desafíos que la ciudad enfrenta y las posibles soluciones que se deben priorizar.

\subsubsection{Articulación y transferencia de capacidades}

Finalmente, la dimensión de articulación y transferencia de capacidades del Laboratorio se traza el objetivo de acompañar a nivel interinstitucional a las diferentes dependencias de la Administración municipal corresponsables del diseño de acciones de cultura ciudadana y cambio comportamental. Así, el Laboratorio es el encargado, desde la gestión del conocimiento, de orientar, validar y acompañar metodológicamente a los equipos de trabajo de la Alcaldía en el diseño de estrategias de intervención territorial en clave de cultura ciudadana.

\subsubsection{Aprendizajes del Laboratorio de Cultura Ciudadana}

El Laboratorio de Cultura Ciudadana como plataforma institucional ha permitido e impulsado nuevas formas de responder a los retos de la organización social, económica y ciudadana de Medellín, consolidándose en sus tres años de implementación en una iniciativa y a la vez en un proceso de coordinación de actores públicos, privados y sociales, que pone en evidencia la oportunidad que representa el diálogo permanente entre el sector público y otros sectores para ampliar las capacidades del estado en la solución de los problemas públicos y orientar el cambio social.

Desde las aproximaciones teóricas y metodológicas del Laboratorio de Cultura Ciudadana, se ha logrado posicionar en la agenda pública la cultura ciudadana y la gobernanza, y con ellas la necesidad que debe integrar en su hacer el gobierno local por entablar un diálogo permanente con la ciudadanía y sus preocupaciones cotidianas, así como buscar respuestas a sus demandas a partir de la colaboración en red con múltiples actores. El Laboratorio como iniciativa, ha permitido que la coordinación institucional y la articulación de actores para la solución de problemas públicos sean procesos cada vez más espontáneos, en el marco de la interacción que tienen las instituciones públicas y privadas con el ciudadano en el espacio público.

El Laboratorio de Cultura Ciudadana de Medellín se ha consolidado como un ecosistema de innovación capaz de integrar a los servidores públicos, la academia, el sector privado y las organizaciones sociales de manera transversal y multidisciplinar, en el diseño de servicios ciudadanos, la identificación de buenas prácticas y la creación de experiencias para aportar soluciones innovadoras en la gestión pública. Así mismo, el respaldo de la academia le ha permitido construir en paralelo una visión holística sobre el futuro de la ciudad y avanzar en 
el diseño de una ruta metodológica que lo ha posicionado como un escenario que cualifica la toma de decisiones de actores públicos, fortalece sus herramientas de gestión y propicia la construcción de acuerdos colaborativos para impulsar transformaciones cotidianas.

Estas fortalezas del Laboratorio no están exentas de retos. Si bien el Laboratorio ha sido un espacio para el diálogo y el encuentro con la ciudadanía, tiene desafíos importantes por avanzar en su sostenibilidad. Una iniciativa como ésta, en el largo plazo, requiere del reconocimiento y la apropiación de la ciudadanía sobre su valor e impacto en la vida cotidiana sin depender del impulso decisional y económico del gobierno. Un ciudadano que participe, construya las experiencias y metodologías, y replique en enfoque mediador de la cultura ciudadana en su entorno microterritorial, de modo tal que asegure la proyección de la iniciativa.

\subsection{Normas sociales y transformaciones culturales: estrategia Ciudadanos como Vos}

Ciudadanos Como Vos es una estrategia de transformación cultural de la Alcaldía de Medellín, implementada desde abril de 2018, tiene como objetivo mejorar la percepción colectiva que se construye en grupos de referencia a través de la generación de escenarios de reconocimiento social y ejercicios de construcción de ciudadanía y convivencia (López et al., 2018). Su trabajo se centra en la implementación de mensajes, actividades y escenarios de encuentro que reproducen imaginarios positivos contribuyendo a reducir la brecha representacional conocida como ignorancia pluralista (Prentice \& Miller, 1996).

De acuerdo con la teoría de las normas sociales (Bicchieri, 2018) el comportamiento individual está definido según las expectativas colectivas (empíricas y normativas), por eso la estrategia supone que la confianza es fundamental para la cultura ciudadana pues ayuda a cerrar las brechas de las percepciones negativas sobre las motivaciones de los otros. Por supuesto, Ciudadanos como vos reconoce la potencia de mecanismos como el agradecimiento, la visibilización de lo cotidiano y la ejemplificación indirecta de lo prosocial para inspirar cambios de comportamiento colectivo.

A través de informar y agradecer, se busca generar un círculo virtuoso en el cual cada vez más ciudadanos se reconozcan como Ciudadanos Como Vos, esto es, parte del grupo de referencia con expectativas de comportamiento particulares. De igual forma, motiva la autorregulación y la visibilización de los cumplidores como mecanismo de replicación (Güiemes \& Wences, 2018). En ese sentido, la estrategia pretende instalar un imaginario de comportamientos prosociales en Medellín, sustentado en aquellos ciudadanos que en su vida diaria están dispuestos a contribuir con la convivencia y la cultura ciudadana. La propuesta es que las personas se reconozcan en esa ciudad que a diario construye convivencia y alejarse de la evaluación maniquea de la ciudadanía. La identificación social hace posible la influencia social y la "interiorización o aceptación personal de las normas" (Güemes \& Wences, 2018, p. 19).

Ciudadanos como vos estableció dos reglas de juego sobre sus intervenciones, que influye en cualquier mensaje o acción asociado a la estrategia. La primera es que el reconocimiento positivo es el enfoque de regulación del comportamiento de los ciudadanos. Esto se traduce en una preocupación por buscar, identificar, involucrar, reconocer y dar visibilidad a los ciudadanos y sus acciones y disposiciones prosociales (López et al., 2018). La segunda es igualar las expectativas y acciones ciudadanas y normalizar lo poderoso de estas acciones, teniendo en cuenta que esas acciones y disposiciones prosociales no son extraordinarias, aunque sí destacables. En este sentido, el conductor de bus que no tiene multas de tráfico o la señora a quien sus vecinos encargan todos los días las llaves de su casa mientras se ausentan de sus viviendas, no son en términos efectivos figuras "heroicas"; son, por el contrario, personas comunes en Medellín y, en efecto, son Ciudadanos como vos. Y de esa "normalidad", de lo común, se infiere la expectativa comportamental.

La estrategia tiene como base seis ejes temáticos correspondientes a los principales retos de convivencia y cultura ciudadana, identificados a partir de diferentes estudios y mediciones, entre ellos la Encuesta de Cultura Ciudadana (Alcaldía de Medellín \& Corpovisionarios, 2017). Estos ejes son: (1) Movilidad segura y sostenible, (2) Convivencia y espacio público, (3) Legalidad y cultura tributaria, (4) Basuras y disposición de residuos, (5) Transformación cultural, (6) Sostenibilidad y medio ambiente. Ciudadanos como vos ha sido estructurada en tres tácticas con objetivos y actividades particulares: (1) Apropiación Ciudadana, (2) Comunicación Pública y (3) Movilización territorial (López et al., 2018). 
En este sentido, vale la pena revisar la propuesta de mensajes usado en la táctica de comunicación pública. La estrategia ha buscado hablar de los ciudadanos como un conjunto incluyente que contribuye con sus acciones cotidianas a la cultura ciudadana y a la convivencia en Medellín. Para la visibilización de comportamientos la estrategia ha hecho uso de datos $\mathrm{y}$ de historias, que muestran a los ciudadanos la cotidianidad de los comportamientos que se quieren promover. En el caso de los datos, se han construido mensajes, basados en evidencia (mediciones, encuestas, estudios) que apelan a la contribución de los ciudadanos a la cultura ciudadana y a la convivencia y la frecuencia con que ciertas cosas ocurren en Medellín. Es decir, que abordan las expectativas empíricas que tienen sobre las normas sociales de la ciudad. Así, los mensajes usados por diferentes canales de comunicación les cuentan a las personas que "En Medellín, 9 de cada 10 ciudadanos se sienten orgullosos de la ciudad", "En Medellín, 8 de cada 10 ciudadanos prefieren dialogar para resolver un conflicto" o "En Medellín, 7 de cada 10 ciudadanos se movilizan en transporte público". Todos estos mensajes se basan en una fuente confiable y actual de información, la Encuesta de Cultura Ciudadana de Medellín 2017.

Las acciones pedagógicas en las calles y barrios de la ciudad también han sido clave en el desarrollo de la estrategia. En el esfuerzo constante por encontrar maneras de agradecer a los ciudadanos cuando tienen comportamientos que contribuyen a la cultura ciudadana, la estrategia ha entregado miles de "FotoCultas" y "Cultas", reconocimientos positivos, y en términos efectivos, contrarios a los comparendos que se imponen a los conductores, que les agradecen por parquearse en los lugares indicados, dar paso a un peatón en un cruce peatonal sin semáforo, dar paso en una congestión vehicular a otro vehículo, entre otros.

Finalmente, la estrategia ha usado los experimentos sociales como mecanismo para "poner en evidencia" las disposiciones prosociales de los ciudadanos de Medellín. En este sentido, se desarrollaron las Tiendas de la Confianza y el Bus de la Confianza (El Tiempo, 2019). Las primeras fueron unas quinientas "tiendas" que vendían productos locales en un sistema de autoservicio en donde cada comprador tomaba sus productos, pagaba y se devolvía dinero sin ningún control o supervisión. El resultado de recaudo de las tiendas era luego comunicado al resto de la ciudad. En el experimento participaron varios miles de personas y el porcentaje de pago acumulado fue del $97 \%$. En el segundo, era un Bus en donde el cobro del ticket se pagaba en un sistema similar al de las tiendas, sin control o supervisión. El bus hacía su ruta normal por la ciudad y transportó en varios días a más de mil cuatrocientos pasajeros, manteniendo un porcentaje de pago de los tickets del $100 \%$. De nuevo, el resultado fue comunicado a toda la ciudad como resultado de otro experimento social colectivo.

\subsubsection{Aprendizajes en Ciudadanos como Vos}

La implementación de la estrategia Ciudadanos como Vos ha permitido reconocer la importancia que los ciudadanos dan a los lenguajes positivos y las oportunidades de vinculación en ejercicios de comunicación pública y programas de cultura ciudadana que adelanta un gobierno. En efecto, la retroalimentación de los ciudadanos con la estrategia evidencia una trayectoria de construcción de confianza, inclusión en los mensajes y apropiación de sus lenguajes. Así, la apuesta por reforzar normas sociales puede nutrirse mucho del uso de mecanismos de reconocimiento como el agradecimiento y la visibilización, usar recompensas simbólicas asociadas a comportamientos que se quieren promover, pero sobre todo su posterior comunicación al resto del grupo de referencia para reforzar las expectativas empíricas del resto (Bicchieri, 2018). Los gobiernos u organizaciones que quieran hacer uso de mecanismos de reconocimiento bien podrían hacer uso de estos incentivos simbólicos en los que la recompensa para el individuo no es material y los receptores principales del mensaje son los terceros que hacen parte de su grupo de referencia (López et al., 2018). Este mecanismo también resulta relevante para contextos en los que la información sobre el comportamiento como norma social sea deficiente, es decir, cuando efectivamente sea difícil evidenciar que un comportamiento responde a una norma social. De igual manera, la implementación de Ciudadanos como Vos ha puesto de manifiesto la relevancia de la preocupación por la confiabilidad de los mensajes, datos e ideas a comunicar y la coherencia en las actividades propuestas. Esto busca, por un lado, evitar o reducir al máximo el escepticismo frente a la información, historias y propuestas presentadas por el gobierno, pero también, la posibilidad de entablar diálogos con los ciudadanos sobre las realidades comunitarias y las oportunidades que presentan para la transformación cultural. El 
capital prosocial que se encuentra en las interacciones más cotidianas puede estar esperando ser puesto en función de resolver algunos problemas de convivencia más complejos.

\section{Conclusión}

La experiencia en intervenciones públicas en Medellín para el cambio de comportamiento, ha dejado un aprendizaje notable en materia de innovación pública. En la medida en que se disponga de espacios de comunicación entre funcionarios públicos, ciudadanía y experticia en materia de investigación e intervención, es posible esperar un nivel sobresaliente de eficacia. Estos espacios de comunicación, en el presente caso, se enfocan en la cultura ciudadana y la gobernanza colaborativa. El principal obstáculo y a la vez el principal recurso para generar cambios en materia de confianza y cumplimiento de acuerdos es el comportamiento humano que se expresa en acciones públicas cotidianas.
La movilidad, el seguimiento de normas de tráfico, el cumplimiento de pago de impuestos, los comportamientos éticos con garantías de transparencia, la disposición a obedecer la ley y las normas sociales que favorecen la cohesión social, son algunos de los ámbitos de estudio e intervención desde esta perspectiva. El Laboratorio de Cultura Ciudadana y Ciudadanos como Vos representan un par de experiencias que ponen en el centro del debate público el cambio de comportamiento y la cultura ciudadana. Un par de experiencias que pueden compararse con otras estrategias similares, implementarse y evaluarse en otros escenarios políticos y de intervención pública a nivel internacional.

\section{Referencias}

Alcaldía de Medellín \& Corpovisionarios. (2017). Diez años construyendo juntos Cultura Ciudadana en Medellín Encuesta de Cultura Ciudadana 2017. Disponible en: https://goo.gl/Mo3QiW (consultado el 21 de octubre de 2018).

Ansell, C., \& Gash, A. (2007). Collaborative Governance in Theory. Journal of Public Administration Research and Theory, 543-571. DOI: https://doi.org/10.1093/jopart/mum032

Asch, S. (1995). Opinions and social pressure. En E. Aronson (Ed.,), Readings about the social animal. Nueva York: W. H Freeman.

Bicchieri, C. (2018). Nadar en contra la corriente. Cómo unos pocos pueden cambiar los comportamientos de toda una sociedad. Bogotá: Editorial Planeta Colombiana S.A.

Bicchieri, C., \& Muldoon, R. (2014). Social norms. Stanford: Encyclopedia of Philosophy.

Echeverri, J., \& Gallego, L. (2019). Cultura ciudadana. Reflexiones y experiencias de ciudad. Medellín: Alcaldía de Medellín - Editorial EAFIT.

Echeverri, J., Gallego, L., \& Silva, S. (2019). Introducción. En: J. Echeverri \& L. Gallego (Eds.), Cultura ciudadana. Reflexiones y experiencias de ciudad. Medellín: Alcaldía de Medellín - Editorial EAFIT.

El Tiempo. (2019). En Medellín rueda el bus en el que la gente paga y toma sus vueltas. Disponible en: https://www.eltiempo.com/colombia/medellin/en-medellin-ya-rueda-el-bus-de-la-confianza-345774 (Consultado el 08/05/2019).

Elster, J. (2010). La explicación del comportamiento social: más tuercas y tornillos para las ciencias sociales. Barcelona: Gedisa.

García Villegas, M. (2017). El orden de la libertad. Bogotá: Fondo de Cultura Económica. 
Grupo Banco Mundial. (2015). Informe sobre Desarrollo Mundial 2015: Mente, sociedad y cultura. Washington D.C.: Banco Mundial.

Güemes, C., \& Wences, S. (2018). Comportamientos incumplidores: contextos y posibles tratamientos políticos. Papers: revista de sociologia, 104(1), 75-99. DOI: http://dx.doi.org/10.5565/rev/papers.2482

Hertwig, R., \& Grüne-Yanof, T. (2017). Nudging and Boosting: Steering or Empowering Good Decisions. Perspectives on Psychological Science, 1-14. DOI: doi.org/10.1177/174569161770249

Kahneman, D. (2012). Pensar rápido, pensar despacio. Bogotá: Debate.

López, N., Silva, S., \& Trujillo, J. (2018). Un juego de espejos: normas sociales, influencia social y cultura ciudadana en Medellín. En: J. Echeverri \& L. Gallego (Eds.), Cultura ciudadana. Reflexiones y experiencias de ciudad. Medellín: Alcaldía de Medellín - Editorial EAFIT.

Murrain, H. (2018). Legalidad y representación del prójimo: un estudio sobre el papel de las normas sociales en el cumplimiento práctico de las leyes. En: C. Tamayo (Ed.), Imaginarios comunes, sueños colectivos y acciones ciudadanas: pensando Medellín en clave de cultura ciudadana, derecho a la ciudad e innovación pública. Medellín: Alcaldía de Medellín - Universidad EAFIT.

Prentice, D. A., \& Miller, D. T. (1996). Pluralistic ignorance and the perpetuation of social norms by unwitting actors. En M. P. Zanna (Ed.), Advances in experimental social psychology, Vol. 28, (pp. 161-209). San Diego, CA, US: Academic Press. http://dx.doi.org/10.1016/S0065-2601(08)60238-5

Sunstein, C. (2017). Paternalismo libertario. Barcelona: Editorial Herder.

Tamayo, C. (2016). Pensar y construir el territorio desde la cultura. Derecho a la ciudad, cultura ciudadana e innovación pública. Medellín: Alcaldía de Medellín - Editorial EAFIT.

Tamayo, C. (2017). Imaginarios comunes, sueños colectivos y acciones ciudadanas: Pensando Medellín en clave de cultura ciudadana, derecho a la ciudad e innovación pública. Medellín: Alcaldía de Medellín Editorial EAFIT.

Thaler, R., \& Sunstein, C. (2017). Un pequeño empujón (Nudges). Bogotá: Taurus.

The Committee for the Prize in Economic Sciences in Memory of Alfred Nobel (2017). Richard H. Thaler: Integrating economics with psychology. Disponible en: https://www.nobelprize.org/nobel_prizes/economicsciences/laureates/2017/advanced.html

Ugarte, Í., Rodríguez, B., Paz, P.M., Páez, D., \& de Lucas, J. (1998). Ignorancia pluralista, atribución de causalidad y sesgos cognitivos en el caso. Revista de Psicología Social, 13(2), 321-330. DOI: https://doi.org/10.1174/021347498760350830 